\title{
A live vaccine for contagious agalactia is protective but does not provoke an ELISA response
}

\author{
Ozdemir $\mathrm{U}^{1}$, Ali Turkyılmaz $\mathbf{M}^{1}$ and Nicholas RAJ ${ }^{2 *}$ \\ ${ }^{1}$ Pendik Veterinary Control Institute, İstanbul, Turkey \\ ${ }^{2}$ The Oaks, Nutshell Lane, Farnham, Surrey, UK
}

\begin{abstract}
This study aimed to assess the efficacy of two Mycoplasma agalactiae vaccines, a live attenuated vaccine (strain AIK) which has been used in the field for over 50 years in Turkey, and an experimental vaccine which was inactivated and adjuvanted with saponin containing the same strain of $M y c o p l a s m a$ agalactiae. A total of 30 Angora goats were assigned to 3 groups, each with 10 animals. Group 1 was inoculated with the attenuated vaccine, group 2 with the inactivated vaccine and group 3

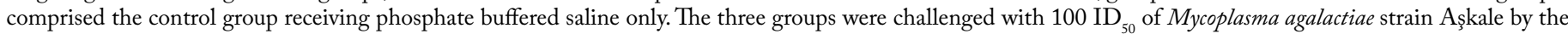
subcutaneous route one month after vaccination. Clinical signs were recorded after challenge and all goats were bled at 14, 21, 28, 42 and 64 days post-vaccination; serological responses to Mycoplasma agalactiae were measured using a commercial ELISA kit. All animals were killed at one month after challenge and internal organs were subjected to bacteriological examination to assess the invasion of the challenge strain. The inactivated vaccine stimulated a strong antibody response as detected by ELISA, whereas the attenuated vaccine did not induce any detectable antibody titres. The challenge strain was re-isolated from internal organs of all the controls and two goats in each vaccinated group. The vaccination-challenge results showed that both vaccines have high efficacy against Mycoplasma agalactiae and that they could be used to control the spread of Mycoplasma agalactiae infection.
\end{abstract}

\section{Introduction}

Contagious agalactia (CA), an important disease of small ruminants, presents mainly as mastitis in lactating animals; it can also cause arthritis, keratoconjunctivitis pneumonia, septicaemia and abscesses in all age groups and abortions in pregnant females [1,2]. The disease leads to significant economic losses because of the reduction in milk yield, death of young animals, abortion and treatment costs [3]. Although the major aetiological agent is Mycoplasma agalactiae (Ma), Mycoplasma capricolum subsp. capricolum, Mycoplasma mycoides subsp. capri and Mycoplasma putrefaciens are also responsible sporadically for a clinically similar syndrome [2]. The disease occurs worldwide, in particular in countries surrounding the Mediterranean and in the Middle East where traditional husbandry is often carried out [4].

CA is difficult to control as antibiotics are rarely completely effective [5]. Commercial vaccines are available and used widely and are mostly formalin, heat-inactivated or aluminum hydroxide- or oil-adjuvanted preparations [2,6]. In Turkey both live attenuated and inactivated vaccines have been used many years [7]. In a recent trial of commercial and experimental CA vaccines, the live vaccine made in Turkey was shown to be the most effective [8]. The aim of this study was to compare the efficacies of the live attenuated vaccine with a vaccine inactivated with saponin in a larger trial.

\section{Material and method}

\section{Vaccines}

Live attenuated vaccine: a vaccine was prepared with the $M$. agalactiae strain AIK isolated from a CA outbreak in Turkey in 1968 and attenuated by 40 serial passages in vitro [9].

Inactivated vaccine: a vaccine was prepared with the second passage of $M$. agalactiae strain AIK by inactivating with $2 \mathrm{mg} / \mathrm{ml}$ of saponin as described by [10] and adjuvanted with oil and Falba [9]. Viability tests showed vaccine to be completely inactivated.

Both vaccines are manufactured and marketed by the Pendik Veterinary Control Institute, Istanbul, Turkey.

\section{Challenge strain}

The strain used for challenging the sheep had been isolated in 1982 from a severe outbreak of CA and designated Aşkale. Unlike the live vaccine strain, the challenge strain produced film and spots in mycoplasma medium and haemolysed medium containing $10 \%$ red blood cells.

\section{Animals}

A total of 30 Angora goats were used. The goats were first bled and tested by a commercial ELISA (Institut Pourquier Version: P00400/05) to confirm the absence of $M$. agalactiae antibodies prior to the experiment.

The goats were assigned to 3 groups consisting of 10 animals each. While group 1 was inoculated with $1 \mathrm{ml}$ of the live attenuated vaccine, group 2 was inoculated with the inactivated vaccine by the subcutaneous route. Group 3 was the control group and received only phosphate buffered saline.

${ }^{\star}$ Correspondence to: Nicholas RAJ, The Oaks, Nutshell Lane, Farnham, Surrey, UK, E-mail: robin.a.j.nicholas@gmail.com

Key words: Mycoplasma agalactiae, contagious agalactia, vaccine, goat

Received: March 01, 2019; Accepted: March 08, 2019; Published: March 11, 2019 
The goats in the experimental and control groups were bled at 14, $21,28,42$ and 64 days post-vaccination and serological responses to M. agalactiae were measured by ELISA following the manufacturer's protocol.

The three groups were challenged with $1 \mathrm{ml}$ of inoculum containing $100 \mathrm{ID}_{50}\left(10^{4}\right.$ microorganisms) of $M$. agalactiae strain Aşkale by the subcutaneous route at one month post-vaccination; clinical signs suggestive CA were recorded daily until autopsy.

All animals were killed at one month post-challenge and various organs: kidney, spleen, liver, lung, hearth, intestine, udder, trachea, joints (carpal, tarsal, knee) and lymph nodes (prescapular, inguinal, popliteal, supramammary, mesenterial, hepatic, mediastinal, bronchial, mandibular) were subjected to bacteriological analysis to assess the invasion of the challenge strain [9]). The mycoplasma were reisolated and identified by established methods [11].

The clinical and cultural findings recorded were scored according to the scheme developed in house at Pendik (Table 1)

\section{Result}

\section{Serological results}

Mean ELISA tests results ( $\mathrm{S} / \mathrm{P} \%)$ of the goats in the groups are shown in Figure 1. The inactivated vaccine (group 2) stimulated a strong antibody response reaching a peak on day 21 . The titres ranged between $57 \%$ and $161 \%$ on the same day. However, the attenuated vaccine did not induce any detectable antibody titers.

\section{Clinical and culture findings}

While no clinical signs were seen in the vaccinated groups, lameness (two animals), keratoconjunctivitis (two animals) and mild fever (one animal) were seen in the control group. Clinical monitoring and re-isolation study results are shown in Table 2.

The challenge strain, recognised by its ability to produce film and spots, was re-isolated and identified from a liver of one goat and lymph nodes of one goat in group 1. In group 2, the strain was reisolated and identified from lungs of one goat and lymph nodes of another. In the control group, $M$. agalactiae was reisolated and identified from the various samples of all animals.

Table 1. The scoring system to calculate immunity

\begin{tabular}{|l|c|}
\hline Findings & Score \\
\hline Isolation of mycoplasma from blood culture, (for each animal) & 2 \\
\hline Isolation of mycoplasma from eyes, noses, milk, feces, (for each isolation) & 1 \\
\hline Isolation for each day & 1 \\
\hline Death following severe disease & 8 \\
\hline Death following moderate disease & 4 \\
\hline Isolation of mycoplasma from internal organs, (for each isolation) & 1 \\
\hline Isolation of mycoplasma from joints, (for each isolation) & 2 \\
\hline Isolation of mycoplasma from lymph nodes, (for each isolation) & 1 \\
\hline
\end{tabular}

\section{Calculation:}

Arithmetic means of the scores of vaccinated and control animals are calculated. Per cent immunity is calculated using the formula below. A vaccine batch which has per cent immunity of 50 or above is considered satisfactory.

$$
\begin{array}{ll}
\text { Mean score of controls } & \mathrm{C} \\
\text { Mean score of vaccinated animals } & \mathrm{V} \\
\text { Per cent immunity }=\frac{C-V}{C} \times 100
\end{array}
$$

Figure 1. Mean serological responses of the groups as detected by ELISA

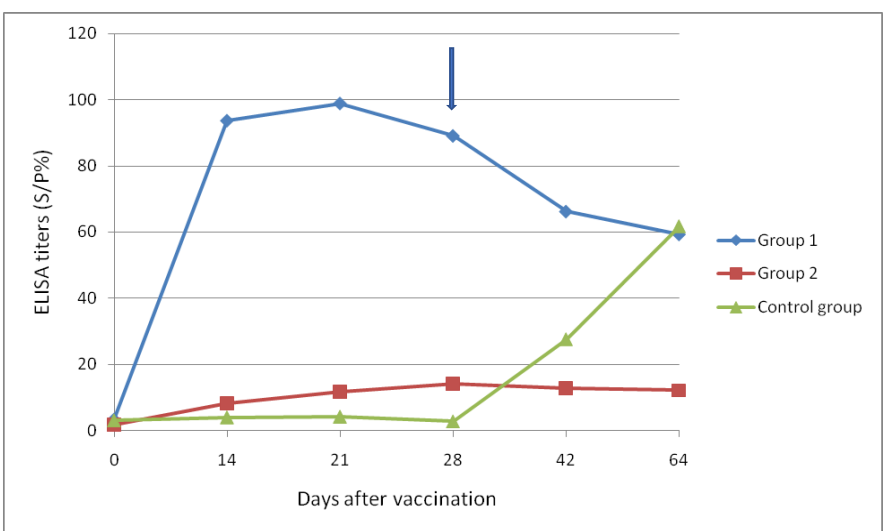

*According to the manufacturer's instructions, $60 \mathrm{~S} / \mathrm{P} \%$ and above should be interpreted as positive.

\begin{tabular}{|c|c|c|c|}
\hline Group & Goat ID & Clinical signs & $\begin{array}{c}\text { Reisolation of } \\
M \text { agalactiae }\end{array}$ \\
\hline \multirow{10}{*}{ Group 1} & 361 & Not seen & $=$ \\
\hline & 366 & Not seen & $=$ \\
\hline & 434 & Not seen & Liver \\
\hline & 435 & Not seen & $=$ \\
\hline & 792 & Not seen & $=$ \\
\hline & 244 & Not seen & $=$ \\
\hline & 253 & Not seen & $=$ \\
\hline & 786 & Not seen & $=$ \\
\hline & 793 & Not seen & $=$ \\
\hline & 784 & Not seen & Lymph node \\
\hline \multirow{10}{*}{ Group 2} & 228 & Not seen & Lung \\
\hline & 315 & Not seen & - \\
\hline & 795 & Not seen & $=$ \\
\hline & 831 & Not seen & $=$ \\
\hline & 832 & Not seen & Lymph node \\
\hline & 801 & Not seen & - \\
\hline & 60 & Not seen & - \\
\hline & 310 & Not seen & - \\
\hline & 451 & Not seen & $=$ \\
\hline & 255 & Not seen & $=$ \\
\hline \multirow{10}{*}{$\begin{array}{c}\text { Group } 3 \\
\text { (Control group) }\end{array}$} & 312 & Not seen & Lymph node \\
\hline & 785 & Mild lameness & $\begin{array}{l}\text { Lymph node, liver, } \\
\text { lung, hearth }\end{array}$ \\
\hline & 836 & keratoconjunctivitis & Lymph node, liver \\
\hline & 63 & Mild fever & Lymph node \\
\hline & 228 & Not seen & Lymph node \\
\hline & 321 & keratoconjunctivitis & Lymph node, lung \\
\hline & 101 & Not seen & Lymph node \\
\hline & 779 & Mild lameness & $\begin{array}{c}\text { Lymph node, liver, } \\
\text { lung }\end{array}$ \\
\hline & 452 & Not seen & Lymph node \\
\hline & 229 & Not seen & Lymph node \\
\hline
\end{tabular}

Arrow signifies time of challenge

Table 2. Clinical and culture findings after the challenge

Based on the scoring system the live and inactivated vaccines studied confer $85 \%$ immunity.

\section{Discussion}

Live Ma vaccines are not permitted in the EU but an attenuated product has been used successfully for many years in Turkey [2,9]. It was produced from the 40th passage of a local Ma strain grown in 
selective media [9]; it retains some pathogenicity for lactating animals which can be further attenuated by another 30 passages but this reduced protection [12]. In the present study, in spite of the lack of a detectable ELISA immune response, animals inoculated with the live attenuated vaccine were largely protected although it must be acknowledged that this was not a severe challenge. Despite this M. agalactiae was reisolated from all unvaccinated sheep.

This failure to generate an antibody response to this vaccine has been reported before [8] and may be due to the random loss of virulence genes during attenuation such as the NIF locus identified by [13]. Mutants lacking this locus also do not provoke an IgG response in experimentally infected ewes though whether the mutants were protective against challenge was not tested.

Alternatively the strains used in the vaccine and ELISA kit may be antigenically disimilar [14] showed extensive molecular diversity amongst $M$. agalactiae strains from several European countries using a range of molecular typing tools. Strains have also shown to be highly variable antigenically which is compounded by their variable surface proteins [15]. This clearly requires further study but this observation can be used to differentiate naturally infected from vaccinated animals in the field [16].

Live vaccines are inexpensive and easy to apply and could have a major impact in areas where disease is endemic. However, they may sometimes cause subclinical infection in lactating females [9] and thus are contra-indicated during this susceptible period. Anecdotal evidence from the field where the live vaccine has been used for many years suggest that they are highly protective with a long duration of immunity (unpublished results). The live vaccine has also been shown to be effective in the face of an outbreak with rapid reductions of clinical signs seen in affected animals similar to that reported recently by [3]. The use of live vaccines in disease-free areas would not be necessary as effective inactivated vaccines described here would be used here.

In a vaccine trial two widely available formalised commercial products were shown to be ineffective against experimental challenge raising concerns for their use in the field [8]. Clearly better vaccines are necessary. [10] compared a range of experimental vaccines. Sheep immunized with saponin- and phenol-inactivated vaccines performed better than those inactivated with formalin, heat and sodium hypochlorite. Our study has confirmed the efficacy of the saponinized inactivated vaccine against $M$. agalactiae producing high levels of immunoglobulin and is protective.

In conclusion this study shows hat both vaccines are highly effective against $M$. agalactiae infection and could be used to control CA by the use of a live vaccine in region where disease is widespread. Ideally, the live vaccines should be part of a regional plan in which all animals likely to come into contact are vaccinated around the same time; an inactivated product would be used in areas of lower disease prevalence and those surrounding infected zones.

\section{Conflict of interest statement}

None of the authors have any conflicting interests.

\section{References}

1. Bergonier D, Berthelot X, Poumarat F (1997) Contagious agalactia of small ruminants: current knowledge concerning epidemiology, diagnosis and control. Rev Sci Tech 16: 848-873. [Crossref]

2. OIE (2008) Contagious agalactia. OIE Terrestrial Manual 2008, OIE, Paris. 992-999.

3. Loria GR, Puleio R, Nicholas RAJ (2018) Contagious Agalactia: Economic Losses and Good Practice. J Bacteriol Mycol 5: 1-3.

4. Loria GR, Nicholas RA (2013) Contagious agalactia: the shepherd's nightmare. Vet $J$ 198: 5-6. [Crossref]

5. Loria GR, Sammartino C, Nicholas RAJ, Ayling RD (2003) In vitro susceptibilities of field isolates of Mycoplasma agalactiae to oxytetracycline, tylosin, enrofloxacin, spiramycin and lincomycin-spectinomycin. Res Vet Sci 75: 3-7. [Crossref]

6. De la Fe C, Assunçao P, Saavedra P, Tola S, Poveda C, et al. (2006) Field trial two dual vaccine against Mycoplasma agalactiae and Mycoplasma mycoides subsp. mycoides (large colony type) in goat. Vaccine 25: 2340-2345. [Crossref]

7. Ozdemir U, Turkaslan T (2003) Mycoplasma strains isolated from contagious agalactia outbreaks. Pendik Vet Microbiol J 34: 33-39.

8. Agnone A, La Manna M, Sireci G, Puleio R, Usticano A, et al. (2013) A comparison of the efficacy of commercial and experimental vaccines for contagious agalactia in sheep. Small Rum Res 112: 230-234.

9. Foggie A, Etheridge JR, Erdag O, Arisoy F (1971) Contagious agalactia of sheep and goats studies on live and dead vaccines in lactating sheep. J Comp Pathol 81: 165-172. [Crossref]

10. Tola S, Manunta D, Roccai S, Rocchigiani AM, Idini G, et al. (1999) Experimental vaccination against Mycoplasma agalactiae using different inactivated vaccine. Vaccine 17: 2764-2768. [Crossref]

11. Nicholas R, Baker S (1998) Methods in molecular medicine, Vol 104: Mycoplasma protocols Ed. R. Miles and R. Nicholas, Humana Pres Inc. 37-51.

12. Arisoy F, Erdag O (1983) Investigation of the immunization and safety of late pregnant and early lactating goats, vaccinated with the attenuated vaccine prepared 69th passage of the Mycoplasma agalactiae (AIK) strain. Pendik Vet Microbiol 31-36.

13. Baranowski E, Bergonier D, Sagne E, Hygonenq MC, Ronsin P, et al. (2014) Experimental infections with Mycoplasma agalactiae identify key factors involved in host-colonisation. PLOS One 9: e93970. [Crossref]

14. McAuliffe L, Churchward CP, Lawes J, Loria GR, Ayling RD, et al. (2019) VNTR analysis reveals unexpected genetic diversity within Mycoplasma agalactiae, the main causative agent of contagious agalactia. BMC Microbiology 8: 193-203.

15. Kumar A, Rahil A, Chakraborty SK, Verma AK, Dhama K (2014) Mycoplasma agalactiae, an Etiological Agent of Contagious Agalactia in Small Ruminants: A Review. Vet Med Int 2014: 286752. [Crossref]

16. Loria G, Puleio R, Agnello S, Marogna G, Nicholas RAJ (2018) Can vaccines for contagious agalactia reduce disease progression in infected sheep: a preliminary study? Vet Record Case Reports 2018-000715.

Copyright: (C2019 Ozdemir U. This is an open-access article distributed under the terms of the Creative Commons Attribution License, which permits unrestricted use, distribution, and reproduction in any medium, provided the original author and source are credited. 